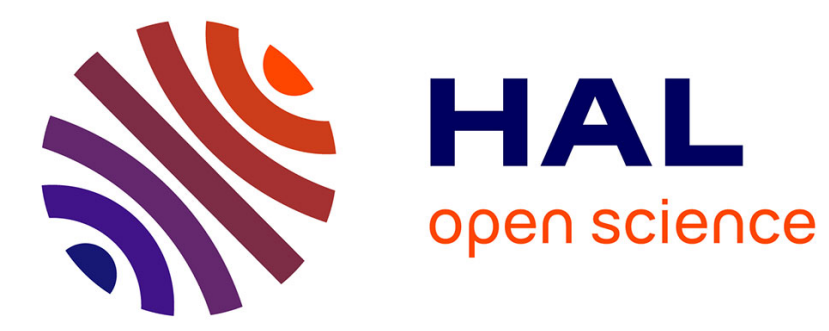

\title{
Observation comparative du déplacement ionique dans les couches minces de PbF2 $\beta$ et de CaF2 par diffusion Rutherford
}

\author{
J.D. Pistre, Y. Danto, J. Salardenne, B. Saboya, J.F. Chemin
}

\section{To cite this version:}

J.D. Pistre, Y. Danto, J. Salardenne, B. Saboya, J.F. Chemin. Observation comparative du déplacement ionique dans les couches minces de PbF2 $\beta$ et de CaF2 par diffusion Rutherford. Revue de Physique Appliquée, 1978, 13 (5), pp.213-218. 10.1051/rphysap:01978001305021300 . jpa-00244442

HAL Id: jpa-00244442

https://hal.science/jpa-00244442

Submitted on 1 Jan 1978

HAL is a multi-disciplinary open access archive for the deposit and dissemination of scientific research documents, whether they are published or not. The documents may come from teaching and research institutions in France or abroad, or from public or private research centers.
L'archive ouverte pluridisciplinaire HAL, est destinée au dépôt et à la diffusion de documents scientifiques de niveau recherche, publiés ou non, émanant des établissements d'enseignement et de recherche français ou étrangers, des laboratoires publics ou privés. 


\title{
REVUE DE PHYSIQUE APPLIQUÉE
}

\author{
Classification \\ Physics Abstracts \\ 66.30-24.50

\section{OBSERVATION COMPARATIVE DU DÉPLACEMENT IONIQUE DANS LES COUCHES MINCES DE $\mathrm{PbF}_{2} \beta$ ET DE $\mathrm{CaF}_{2}$ PAR DIFFUSION RUTHERFORD}

\author{
J. D. PISTRE, Y. DANTO, J. SALARDENNE
}

Laboratoire de Recherches en Electrotechnique et Physique du Solide

\section{B. SABOYA et J. F. CHEMIN}

Centre d'Etudes Nucléaires de Bordeaux-Gradignan (C. E. N. B. G.)

Université de Bordeaux I, Domaine Universitaire, 33405 Talence, France

\begin{abstract}
(Reçu le 13 juillet 1977, révisé les 24 octobre 1977, 23 décembre 1977, 13 janvier 1978,
\end{abstract} accepté le 18 janvier 1978)

\begin{abstract}
Résumé. - Des couches minces de $\mathrm{PbF}_{2} \beta$ et de $\mathrm{CaF}_{2}$, dont les conductivités ioniques sont très différentes, ont été analysées par rétro-diffusion de particules $\alpha$. On a pu observer, dans le cas de $\mathrm{PbF}_{2}$, une variation importante du rapport des concentrations fluor/plomb dans l'épaisseur de la couche, correspondant à une accumulation de fluor du côté du faisceau incident. Cet effet est atténué dans les couches de $\mathrm{CaF}_{2}$. L'interprétation des résultats est basée sur l'existence d'un nombre important de défauts créés par le faisceau, et sur leur déplacement sous l'effet de la charge superficielle due à l'émission secondaire d'électrons.
\end{abstract}

Abstract. - Thin films of $\mathrm{PbF}_{2} \beta$ and $\mathrm{CaF}_{2}$, two fluoride compounds with very different ionic conductivities, have been analysed by backscattering of $\alpha$-particles. For $\mathrm{PbF}_{2}$, an important variation of the fluorine/lead concentration ratios versus the film layer depth has been observed. This result corresponds to a fluorine accumulation towards the external side of the sample. This effect is less important in the case of the $\mathrm{CaF}_{2}$ films. The interpretation of the results is based on the existence of an important number of defects created by the beam, and on their motion under the influence of the surface charge due to the secondary electron emission.

1. Introduction. - La rétrodiffusion de particules $\alpha$ est une méthode maintenant classique d'analyse de la composition et des profils de concentration des éléments du matériau d'une cible [1]. Un travail précédent, effectué par cette technique [2], nous a permis de mettre en évidence dans des couches minces d'un superconducteur ionique, $\mathrm{PbF}_{2} \beta$, un important gradient de concentration du fluor dans l'épaisseur de la cible. Nous avons repris cette étude de manière systématique, sur un grand nombre d'échantillons, et par comparaison avec un matériau de même structure, $\mathrm{CaF}_{2}$, présentant une conductivité ionique très inférieure [3, 4].

Nous pensons ainsi montrer que la différence de comportement entre ces deux matériaux résulte, en partie, de la différence de mobilité des ions et donc que la diffusion Rutherford est un moyen expérimental d'observation du déplacement des espèces mobiles dans les conducteurs ioniques. Pour rendre compte des résultats obtenus, il est nécessaire d'analyser l'influence de différents effets secondaires (émission d'électrons, déplacement d'atomes) dus à l'irradiation du matériau par les particules $\alpha$ du faisceau.
Enfin, pour la détermination des impuretés proprement dites contenues dans chacun des deux matériaux, nous avons utilisé la complémentarité apportée par l'analyse du rayonnement $\mathrm{X}$ émis (réactions $\alpha, \mathrm{X}$ ).

2. Technique expérimentale. - 2.1 Elaboration DES ÉCHANTILLONS. - Les couches minces ont été réalisées par évaporation thermique sous un vide de $10^{-6}$ torr à partir de creusets de molybdène chauffés par effet Joule. La vitesse de condensation a été maintenue constante et fixée à $5 \AA /$ s. Dans ces conditions, la température du creuset ne dépasse pas $730^{\circ} \mathrm{C}$ pour $\mathrm{PbF}_{2} \beta$ et peut atteindre $1100^{\circ} \mathrm{C}$ pour $\mathrm{CaF}_{2}$. Le produit de départ est dans chaque cas de la poudre de haute pureté, refluorée avant utilisation $\left({ }^{1}\right)$. Le substrat qui convient le mieux pour cette étude est le carbone autosupporté de $1000 \AA ̊$ d'épaisseur environ [5], dont une moitié seulement reçoit le dépôt. La deuxième partie sert de référence permettant d'éliminer du spectre obtenu, la contribution du support.

(1) Préparée au Laboratoire de Chimie du Solide de l'Université de Bordeaux I. 
2.2 Méthodes D'ANALYSES. - Rappelons que la perte d'énergie d'une particule chargée dans l'épaisseur d'une cible est due essentiellement à deux types d'interactions auxquelles se rattachent des probabilités différentes selon l'énergie, la masse et l'état de charge de l'ion incident. On observe d'une part l'interaction des particules incidentes avec les couches électroniques des atomes de la cible, ce qui entraîne un effet de ralentissement augmentant avec l'épaisseur du matériau traversé ; d'autre part, par diffusion élastique sur les noyaux de la cible, la particule incidente est diffusée et l'énergie perdue est alors caractéristique de l'atome diffuseur. On a alors :

$$
E_{1}=k^{2} E_{0} \quad \text { avec } \quad k \simeq \frac{M_{1} \operatorname{Cos} \theta_{\mathrm{L}}+M_{2}}{M_{1}+M_{2}}
$$

avec $E_{1}, M_{1}$ énergie et masse de la particule rétrodiffusée, $M_{2}$ masse de l'atome diffuseur, $E_{0}$ énergie de la particule incidente, $\theta_{\mathrm{L}}$ angle de diffusion dans le système du laboratoire.

Il faut noter que (1) est valable quel que soit $\theta_{\mathrm{L}}$ pour $M_{2} / M_{1} \geqslant 4$. Le premier processus d'interaction peut entraîner une ionisation des couches électroniques profondes suivies de l'émission de raies $\mathrm{X}$ caractéristiques des éléments constituant le matériau. Les sections efficaces d'ionisation sont à l'heure actuelle bien connues $[6,7,8]$. La diffusion Rutherford permet elle aussi d'effectuer le dosage et en plus de déterminer l'épaisseur et la stœchiométrie de la cible étudiée. En effet, la perte d'énergie de la particule incidente dans l'épaisseur de la cible avant et après diffusion entraîne un déplacement ou un élargissement des pics selon l'épaisseur de cible traversée, ce qui permet dans la plupart des cas de préciser la position des éléments dans la couche et même d'établir un profil de concentration en fonction de la profondeur. Ces méthodes constituent désormais des moyens classiques de caractérisation des couches minces $[9,5]$.

La stœchiométrie de la couche à une épaisseur comprise entre $e$ et $(e+\mathrm{d} e)$ s'établit à partir de la relation $[5,9]$ :

$$
[S]_{e}=\left[\frac{N_{2}}{N_{1}}\right]_{e}=\left[\frac{Z_{1}}{Z_{2}}\right]^{2}\left[\frac{n_{2}}{n_{1}}\right]_{e}
$$

$N_{1}$ et $N_{2}$ représentent la concentration en atomes d'espèces 1 et $2, Z_{1}$ et $Z_{2}$ étant les numéros atomiques de ces atomes. $n_{1}$ et $n_{2}$ correspondent aux nombres de particules détectées, après diffusion par les noyaux des atomes 1 et 2 respectivement, à une profondeur comprise entre $e$ et $(e+\mathrm{d} e)$ repérée à partir de leur perte d'énergie dans le matériau.

Complémentarité des deux méthodes d'analyse [10]. La détection par réaction $\alpha-\mathrm{X}$ a une sensibilité d'autant meilleure que le numéro atomique de l'élément détecté est faible. La situation est inversée dans le cas de la diffusion Rutherford. De plus, dans le cas de cette seconde méthode, le pouvoir de séparation diminue aux fortes masses, ce qui, ajouté aux déplacements et à l'élargissement des pics rend difficile une identification de chaque élément. Les réactions $\alpha$-X permettent alors une identification plus sûre. L'énergie des particules incidentes fixée à $2,5 \mathrm{MeV}$ a été choisie de façon à se placer dans des conditions de sections efficaces optimales pour la plupart des éléments analysés.

2.3 EFFETS SECONDAIRES. - L'irradiation du matériau de la cible par des particules $\alpha$ de haute énergie entraîne l'apparition d'un certain nombre d'effets secondaires parmi lesquels la création de défauts par déplacement d'atomes ou d'ions et l'émission d'électrons.

Au début de l'irradiation, dans le cas où la cible est un électrolyte solide, le déplacement d'ions conduit à un accroissement du nombre de défauts et par suite à une augmentation de la conductivité ; parallèlement, le désordre créé par le rayonnement a pour effet de diminuer progressivement les mobilités des espèces ioniques. Par la suite, lorsque le désordre est devenu très grand, les contributions relatives des différents mécanismes au transport peuvent être complètement modifiées.

En ce qui concerne l'émission d'électrons secondaires, il a été montré expérimentalement que l'épaisseur du matériau pour laquelle les électrons sont extraits de la cible, est limitée (de l'ordre de la centaine d'angströms) [11, 12]. Dans ces conditions, si le matériau de la cible est un isolant, le faisceau incident des particules $\alpha$ est responsable de l'apparition d'une charge superficielle positive. Il en résulte, si le matériau est faiblement conducteur, un équilibre dynamique entre la vitesse de renouvellement de cette charge fixe et le flux des charges mobiles susceptibles de traverser le matériau pour la neutraliser. En particulier, lorsque le matériau est un conducteur ionique, ces charges mobiles seront constituées par les défauts ponctuels déjà présents ou créés par le rayonnement ainsi que par les électrons excités.

2.4 CONDITIONS EXPÉRIMENTALES. - Nous avons utilisé le faisceau de particules $\alpha$ de 2,5 MV produit, avec une intensité de l'ordre du nA pour une section d'impact d'environ $1 \mathrm{~mm}^{2}$, par l'accélérateur Van de Graaff de $4 \mathrm{MV}$ du C. E. N. B. G. La détection des particules rétrodiffusées est effectuée à l'aide d'un détecteur à barrière de surface dont la résolution mesurée avec une source ${ }^{241} \mathrm{Am}$ est de $13,5 \mathrm{keV}$. Le rayonnement $\mathrm{X}$ est détecté par une diode $\mathrm{Si}(\mathrm{Li})$ dont la résolution est de $217 \mathrm{eV}$ pour les raies d'énergie $5,9 \mathrm{keV}$. Les résultats stockés dans un analyseur 4096 canaux sont transférés sur un calculateur PDP 15 pour la détermination des taux d'impuretés et des profils de concentration.

3. Résultats. - Nous avons montré lors d'une étude précédente [2] que les couches de fluorure de plomb sont très bien cristallisées, l'axe $\langle 111\rangle$ de la maille fluorine étant perpendiculaire au substrat. Un résultat analogue a été observé pour les couches de $\mathrm{CaF}_{2}$. 


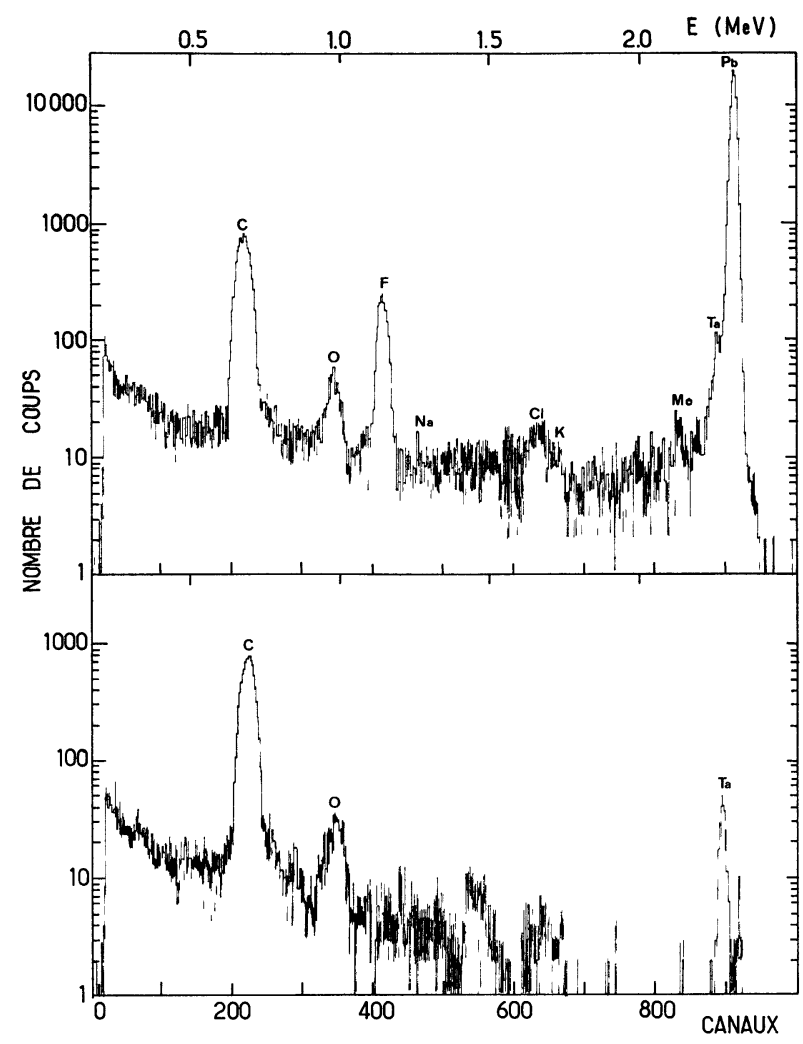

Fig. 1a. - Spectre de diffusion Rutherford obtenu pour une couche de $\mathrm{PbF}_{2}$ de $50 \AA$ déposée sur carbone mince. Le spectre inférieur correspond au support seul.

[Rutherford backscattering spectrum got from a $\mathrm{PbF}_{2}$ film of $50 \AA$ deposited on thin carbon. The lower spectrum is relative to the substrate alone.]

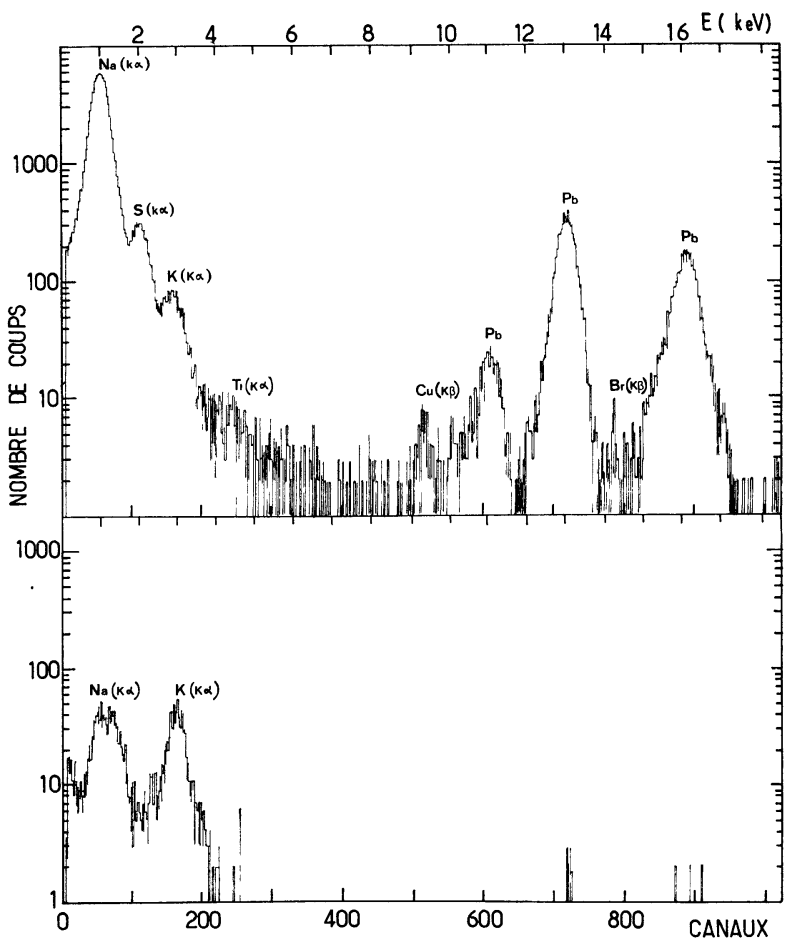

FIG. 1c. - Spectre de réactions $\alpha \mathrm{X}$ obtenu pour une couche de $\mathrm{PbF}_{2}$ de $50 \AA$ déposée sur carbone mince. Le spectre inférieur correspond au support seul.

$\left[\alpha \mathrm{X}\right.$ reactions spectrum got from a $\mathrm{PbF}_{2}$ film of $50 \AA$ deposited on thin carbon. The lower spectrum is relative to the substrate t.

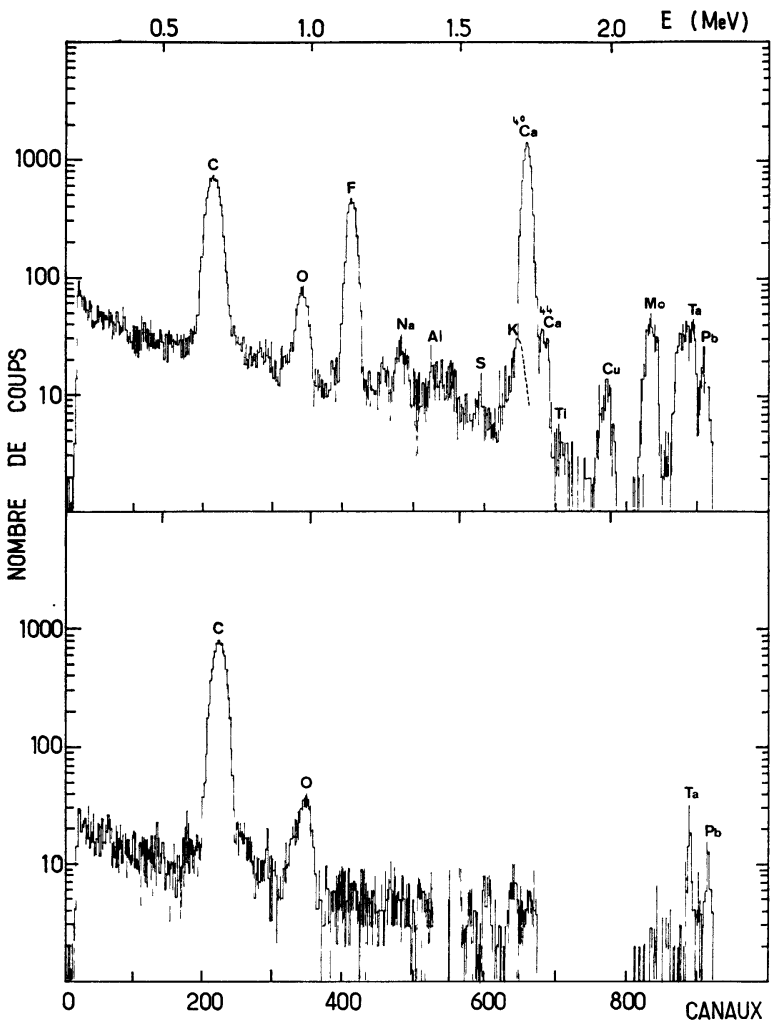

FIG. 1b. - Spectre de diffusion Rutherford obtenu pour une couche de $\mathrm{CaF}_{2}$ de $50 \AA$ déposée sur carbone mince. Le spectre inférieur correspond au support seul.

[Rutherford backscattering spectrum got from a $\mathrm{CaF}_{2}$ film of $50 \AA$ deposited on thin carbon. The lower spectrum is relative to the substrate alone.]

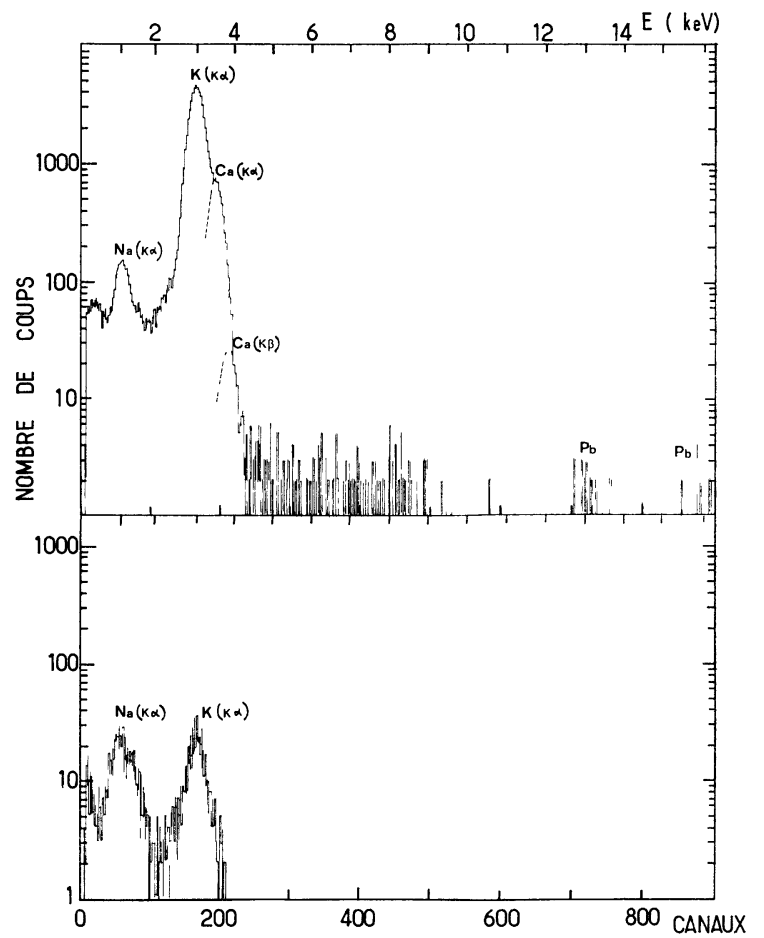

FIG. 1d. - Spectre de réactions $\alpha \mathrm{X}$ obtenu pour une couche de $\mathrm{CaF}_{2}$ de $50 \AA$ déposée sur carbone mince. Le spectre inférieur correspond au support seul.

$\left[\alpha \mathrm{X}\right.$ reactions spectrum got from a $\mathrm{CaF}_{2}$ film of $50 \AA$ deposited on thin carbon. The lower spectrum is relative to the substrate alone.] 


\section{TABleaU I}

Identification et dosage des impuretés contenues dans les couches minces de $\mathrm{PbF}_{2}$ et de $\mathrm{CaF}_{2}$. Nombre d'atomes exprimés en \%o du métal de base

\begin{tabular}{|c|c|c|c|c|c|c|c|c|c|c|}
\hline \multirow[b]{2}{*}{$\begin{array}{l}\text { Nature } \\
\text { de la couche }\end{array}$} & \multirow[b]{2}{*}{ Epaisseur } & \multicolumn{9}{|c|}{ Elément } \\
\hline & & $\mathrm{O}$ & $\mathrm{Na}$ & $\mathrm{Cl}$ & $\mathrm{K}$ & $\mathrm{Ti}$ & $\mathrm{Cu}$ & $\mathrm{Br}$ & Mo & $\mathrm{Sb}$ \\
\hline $\mathrm{PbF}_{2}$ & $50 \AA$ & $\begin{array}{l}52,5 \\
(\mathrm{R})\end{array}$ & $\begin{array}{l}2,2 \\
(X)\end{array}$ & $\begin{array}{l}14 \\
\text { (R) }\end{array}$ & $\begin{array}{r}5,5 \\
(\mathrm{X})\end{array}$ & $\begin{array}{l}3,3 \\
(\mathrm{X})\end{array}$ & $\begin{array}{c}2,9 \\
(\mathrm{X})(\mathrm{R})\end{array}$ & $\begin{array}{c}3,3 \\
(X)(R)\end{array}$ & $\begin{array}{l}4,2 \\
(\mathrm{R})\end{array}$ & $\begin{array}{l}2, \\
(\mathbf{R}\end{array}$ \\
\hline $\mathrm{CaF}_{2}$ & $50 \AA$ & $\begin{array}{l}108 \\
(\mathrm{R})\end{array}$ & $\begin{array}{c}55,1 \\
(\mathrm{X})(\mathrm{R})\end{array}$ & $\begin{array}{r}7,4 \\
(\mathrm{R})\end{array}$ & $\begin{array}{l}22,2 \\
(\mathrm{X})\end{array}$ & $\begin{array}{c}2,2 \\
(\mathrm{X})(\mathrm{R})\end{array}$ & $\begin{array}{l}3,8 \\
(\mathrm{R})\end{array}$ & & $\begin{array}{l}6,0 \\
(\mathrm{R})\end{array}$ & \\
\hline
\end{tabular}

(R) Résultats obtenus en rétrodiffusion.

(X) Résultats obtenus par réaction $(\alpha, X)$.

L'analyse des spectres de diffusion Rutherford et de réactions $\alpha-\mathrm{X}$ (Fig. $1 a, b, c, d$ ) montre que les taux d'impuretés, faibles pour $\mathrm{PbF}_{2}$, sont plus élevés pour $\mathrm{CaF}_{2}$ (tableau I). Cette différence est certainement liée à l'écart des températures de sublimation des deux composés, compte tenu du mécanisme d'évaporation préférentielle des impuretés [13].

L'observation fondamentale que l'on peut faire à partir de ces mesures concerne les profils de concentration des ions fluor. Ces profils ont été observés à $25^{\circ} \mathrm{C}$, l'élévation de température due à l'énergie cédée à la cible par le faisceau étant dans tous les cas négligeable. Les figures $3,4,5$ et 6 donnent les résultats pour des couches minces de $\mathrm{PbF}_{2}(800 \AA$ et $2200 \AA)$ et de $\mathrm{CaF}_{2}(780 \AA$ et $2400 \AA)$. Deux spectres d'énergie à partir desquels ont été établis ces profils sont donnés figures $2 a$ et $2 b$.

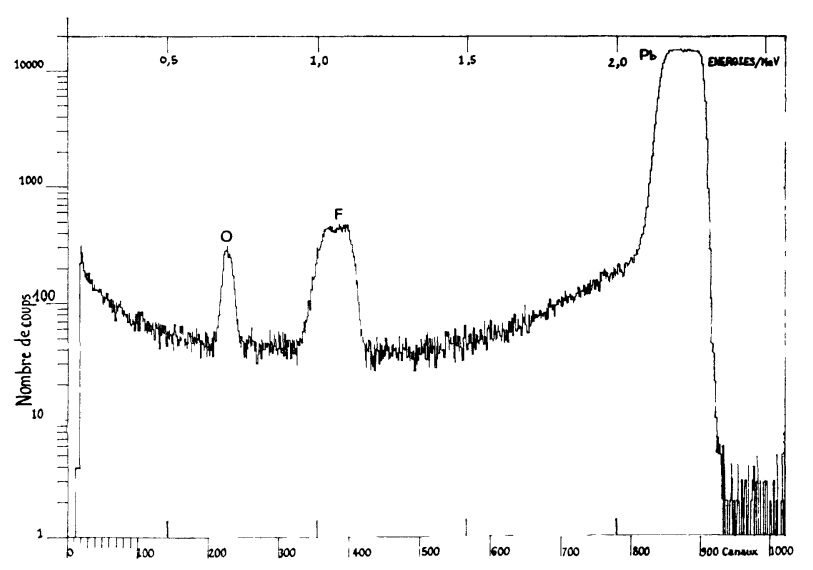

FIG. 2a. - Spectre de diffusion Rutherford obtenu pour une couche de $\mathrm{PbF}_{2}$ de $800 \AA$ déposée sur carbone mince.

[Rutherford backscattering spectrum got from a $\mathrm{PbF}_{2}$ film of $800 \AA$ deposited on thin carbon.]

Dans le cas de $\mathrm{PbF}_{2}$ le rapport $[\mathrm{F}] /[\mathrm{Pb}]$ de la concentration des deux éléments varie considérablement dans l'épaisseur des couches ; il est très faible côté support, pour atteindre dans certains cas des valeurs de l'ordre de 3 côté faisceau incident (Fig. 3 et 5). D'autre part, la zone à forte concentration de fluor s'étend sur une plus

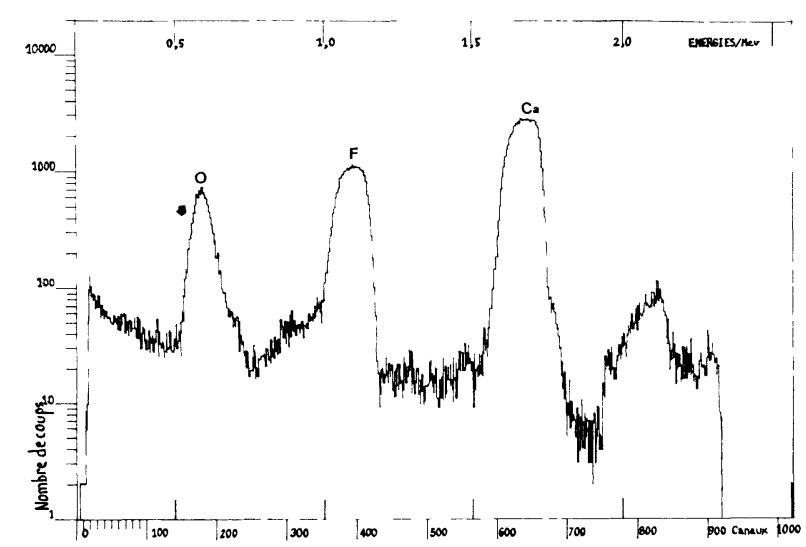

FIG. 2b. - Spectre de diffusion Rutherford obtenu pour une couche de $\mathrm{CaF}_{2}$ de $780 \AA$ déposée sur carbone mince.

[Rutherford backscattering spectrum got from a $\mathrm{CaF}_{2}$ film of $780 \AA$ deposited on thin carbon.]

grande partie de l'épaisseur de la couche que celle à faible concentration. Un profil de concentration ayant une allure analogue, mais très atténuée, est observé pour $\mathrm{CaF}_{2}$ (Fig. 4 et 6).

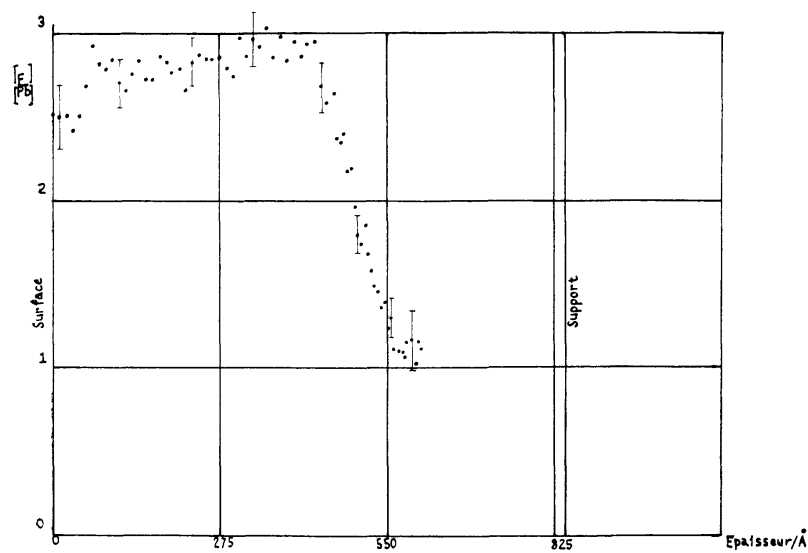

FIG. 3. - Profil de concentration du fluor par rapport au plomb dans une couche mince de $800 \AA$ dont le spectre de rétrodiffusion est donné figure $2 a$.

[Concentration, versus thickness, of fluorine in comparison with lead for a $800 \AA \mathrm{PbF}_{2}$ film which backscattering spectrum is given Fig. 2a] 


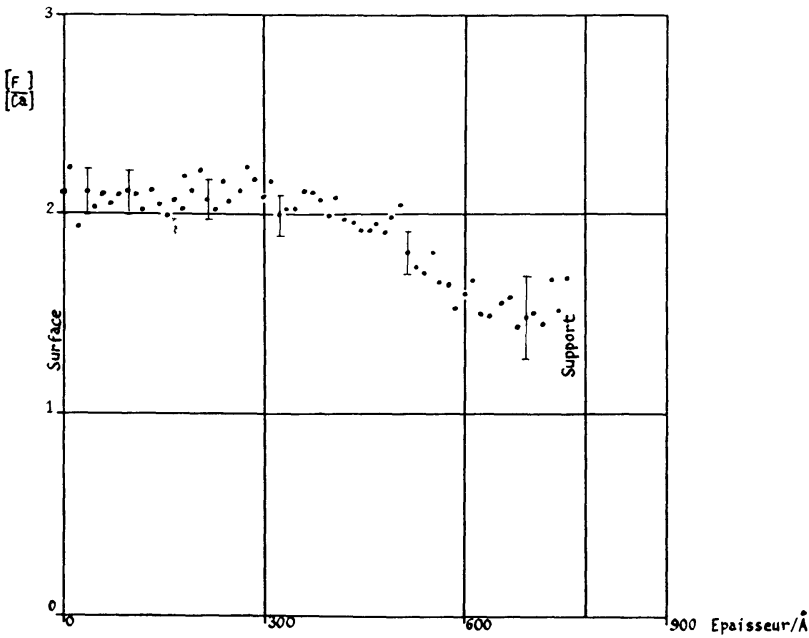

Fig. 4. - Profil de concentration du fluor par rapport au calcium dans une couche mince de $780 \AA$ dont le spectre de rétrodiffusion est donné figure $2 b$.

[Concentration, versus thickness, of fluorine in comparison with calcium for a $780 \AA \mathrm{PbF}_{2}$ film which backscattering spectrum is given Fig. $2 b$ ]

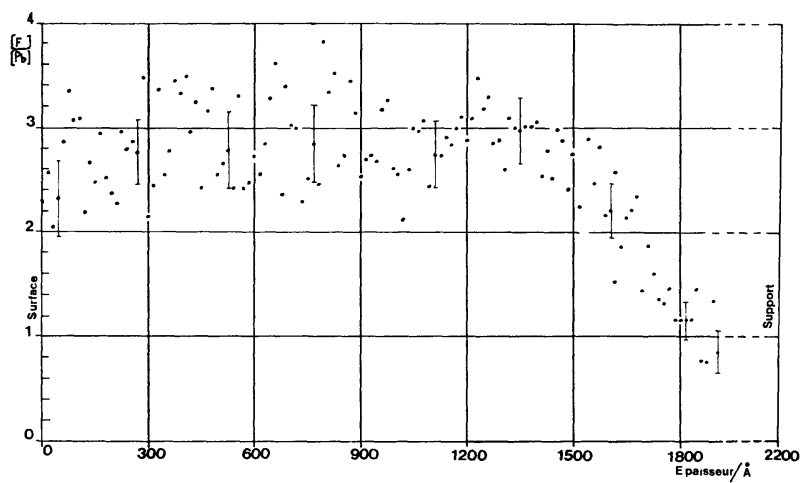

Fig. 5. - Profil de concentration du fluor par rapport au plomb dans une couche mince de $2200 \AA$.

[Concentration, versus thickness, of fluorine in comparison with lead for a $2200 \AA \mathrm{PbF}_{2}$ film]

Le tableau II donne la concentration moyenne de fluor par rapport au plomb ou au calcium pour différentes couches. Ce rapport est voisin de 2 pour le fluorure de plomb. Les couches de fluorure de calcium sont nettement déficitaires en fluor. Enfin, pour des durées d'irradiation variant de $2 \mathrm{mn}$ (temps minimum pour avoir un stockage suffisant) à $20 \mathrm{mn}$, on ne constate pas d'évolution notable des profils de concentration ; seule,

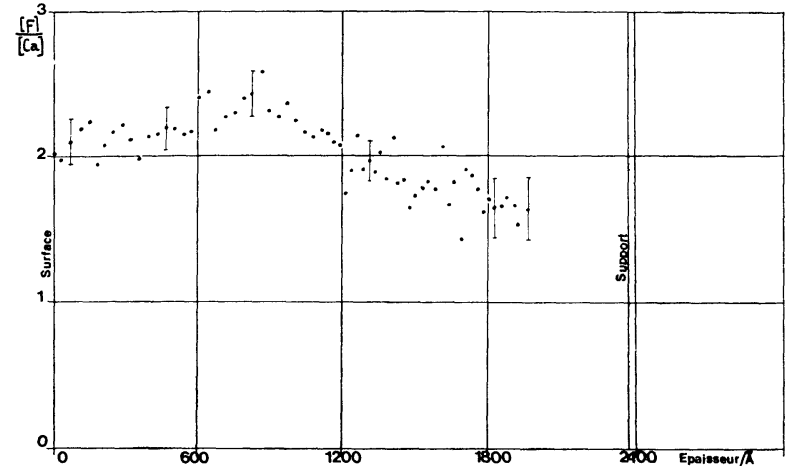

Fig. 6. - Profil de concentration du fluor par rapport au calcium dans une couche mince de $2400 \AA$.

[Concentration, versus thickness, of fluorine in comparison with calcium for a $2400 \AA \mathrm{CaF}_{2}$ film.]

une diminution du rapport des concentrations moyennes $[\mathrm{F}] /[\mathrm{Pb}]$ est observée, comme le montre la figure 7 , obtenue par intégration, à intervalles de temps réguliers, du nombre de coups sous chaque pic.

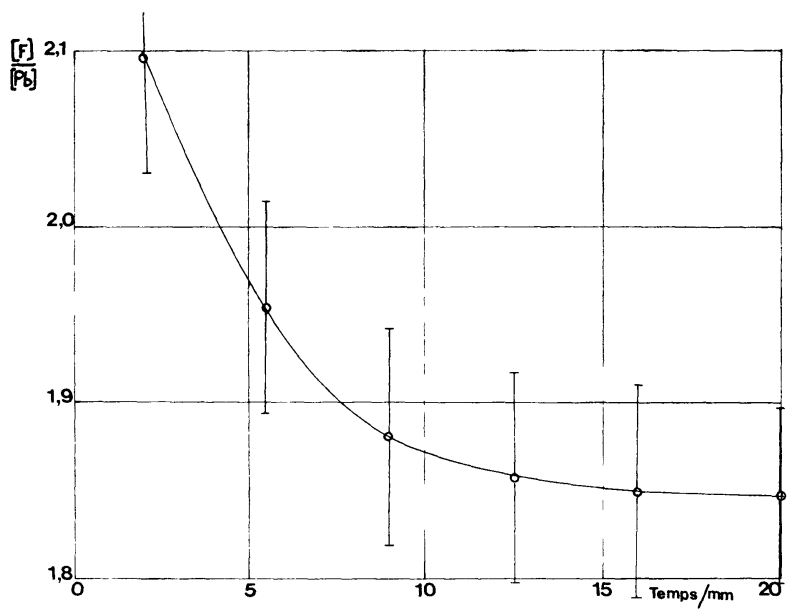

Fig. 7. - Concentration moyenne du fluor par rapport au plomb dans une couche mince de $\mathrm{PbF}_{2}$, en fonction du temps d'irradiation.

[Average concentration, measured versus the irradiation time, of fluorine in comparison with lead for a $500 \AA \mathrm{PbF}_{2}$ film.]

4. Discussion. - L'allure des résultats obtenus peut s'interpréter sur la base des remarques faites au paragraphe 2.3. L'émission secondaire est responsable de l'apparition d'une charge superficielle sur la cible côté faisceau incident. Le champ qui en résulte dans le

TABLEAU II

Concentration moyenne du fluor par rapport au calcium ou au plomb dans des couches minces de $\mathrm{CaF}_{2}$ et de $\mathrm{PbF}_{2}$

Nature de la couche

$\mathrm{PbF}_{2}$

$\mathrm{CaF}_{2}$
50

$1,90 \pm 0,08$

$1,87 \pm 0,09$
780

$1,80 \pm 0,05$
Epaisseur ( $\AA$ )

$\begin{array}{ccc}800 & 2200 & 2400 \\ 2,00 \pm 0,05 & 1,96 \pm 0,04 & -\end{array}$


matériau provoque un déplacement des porteurs mobiles venant neutraliser cette charge. Il s'ensuit un équilibre dynamique. L'observation des profils de concentration décrits ci-dessus conduit aux conclusions suivantes :

4.1 Ces profils résultent d'un déplacement des espèces ioniques mobiles. Ils ne peuvent s'expliquer que si la concentration des défauts présents est beaucoup plus élevée que celle correspondant à l'équilibre thermodynamique (estimée à $10^{16} \mathrm{~cm}^{-3}$ [14]). Le comportement de $\mathrm{PbF}_{2}$, caractérisé par une accumulation des ions fluor du côté de la surface externe, plus forte que pour $\mathrm{CaF}_{2}$, peut être interprété :

- par sa conductivité ionique plus importante $\left(4 \times 10^{-4} \Omega^{-1} \cdot \mathrm{cm}^{-1}[4]\right.$ contre $2 \times 10^{-9} \Omega^{-1} \cdot \mathrm{cm}^{-1}$ pour $\mathrm{CaF}_{2}$ à la même température de $500 \mathrm{~K}$ [3]) ; - par la possibilité d'ionisation du plomb à l'état $\mathrm{Pb}^{4+}$, permettant d'envisager la formation d'une solution solide du type $\mathrm{PbF}_{2+} X$.

4.2 La zone d'accumulation des lacunes de fluor côté substrat s'étend sur une épaisseur relativement faible par rapport à la zone d'accumulation des anions. Ceci peut s'interpréter si l'on admet une mobilité des lacunes très supérieure à celle des anions, ce qui rejoint les conclusions obtenues dans des conditions différentes par d'autres auteurs [14, 15 et 16].

4.3 En un temps relativement court (inférieur à $2 \mathrm{mn}$ ) le déplacement d'atomes sous l'effet du rayonnement à l'intérieur de la structure entraîne un désordre tel que la mobilité des espèces ioniques devient très faible. Dès lors, les spectres obtenus se modifient très peu dans le temps ; le courant de neutralisation de la charge superficielle est devenu essentiellement le fait des électrons excités.

4.4 La baisse de concentration des ions fluor observée sur une faible épaisseur, côté surface irradiée, résulte vraisemblablement d'un arrachement de ces ions par les particules incidentes. Les résultats obtenus figure 7 où apparaît une diminution progressive au cours du temps de la concentration moyenne du fluor par rapport au plomb, laissent supposer qu'il subsiste une migration lente des ions $\mathrm{F}^{-}$vers la surface à partir de laquelle ils sont arrachés.

5. Conclusion. - Nous avons pu observer, dans le cas de couches minces de $\mathrm{PbF}_{2} \beta$ déposées sur du carbone, une variation importante du rapport de la concentration des atomes de fluor par rapport à la concentration des atomes de plomb dans l'épaisseur de la couche. Un profil, d'ailleurs analogue, est également observé pour des couches de $\mathrm{CaF}_{2}$ mais très atténué. On peut rendre compte de ces résultats en admettant un taux élevé de création de défauts par le faisceau et un déplacement de ces défauts sous l'effet de la charge superficielle due à l'émission secondaire d'électrons. La différence de comportement entre $\mathrm{PbF}_{2}$ et $\mathrm{CaF}_{2}$ montre que le rapport des mobilités ioniques dans ces deux matériaux est conservé, lorsqu'ils sont soumis au bombardement par le faisceau, au moins pendant les premiers instants de l'irradiation. En ce qui concerne le $\mathrm{PbF}_{2} \beta$, de même, tant que la structure n'est pas trop perturbée, la mobilité des lacunes d'anions reste très supérieure à celle des interstitiels conformément aux résultats connus pour ce matériau [14]. Par la suite, le désordre créé par le rayonnement est tel que le mouvement des charges électroniques devient prépondérant dans le matériau cible ; une lente diminution en fonction du temps du rapport moyen $[\mathrm{F}] /[\mathrm{Pb}]$ est constatée, qui peut s'interpréter par une dérive très lente des anions vers la surface externe de la couche, où ils sont arrachés.

$\mathrm{Au}$ total, l'étude de superconducteurs ioniques par rétrodiffusion de particules $\alpha$, doit permettre d'obtenir des informations à la fois sur les mécanismes de déplacement des espèces mobiles et sur les taux de création de défauts par le faisceau dans ces matériaux.

\section{Bibliographie}

[1] Davies, J. A., Mayer, J. W. and Ericsson, L., Can. J. Phys. 46 (1968) 663.

[2] Pistre, J. D., Salardenne, J. and Smutek, P., Electrochim. Acta 22 (1977) 693.

[3] Ure, R. W., J. Chem. Phys. 26 (1957) 1363.

[4] Schoonman, J., Dirksen, G. J. and Blasse, G., J. Solid State Chem. 7 (1973) 245.

[5] Saboya, B., Chemin, J. F., Roturier, J., Barrière, A. S., Danto, Y. and Salardenne, J., J. Phys. D : Appl. Phys. 8 (1975) 1008.

[6] Rutledge, C. H., WAtson, R. L., Atomic and Nuclear Data Tables 12 (1973) 195.

[7] HARDt, T. L., WAtson, R. L., Atomic and Nuclear Data Tables 17 (1976) 107.

[8] Chemin, J. F., Mitchell, I. V. and Saris, F. W., J. Appl. Phys. Part I and II 45 (1974) 532.
[9] Mackintosh, W. D., « The characterization of solid surfaces by Rutherford Scattering » (Rapport interne) - Chalk River Ontario (1972).

[10] Saboya, B., Thèse de $3^{\mathrm{e}}$ cycle, Université de Bordeaux I, 33405 Talence, France.

[11] Hill, A. G., Buechner, W. W., Clark, J. S. and FisK, J. B., Phys. Rev. 55 (1939) 463.

[12] Carter, G. and Colligon, J. S., Ion Bombardment of Solids (Heinemann Educational Books - London).

[13] Salardenne, J., Danto, Y. and Barrière, A. S., Ann. Chimie 10 (1975) 201.

[14] Bonne, R. W. Schoonman, J., J. Electrochem. Soc. 124 (1977) 28.

[15] Liang, C. C. and Joshi, A. V., J. Electrochem. Soc. 122 (1975) 466.

[16] Kennedy, J. H. and Miles, R. C., J. Electrochem. Soc. 123 (1976) 47. 